\title{
WORKER MOVEMENT DIAGRAM BASED STOCHASTIC MODEL OF OPEN PACED CONVEYORS
}

\author{
TAMÁS RUPPERT ${ }^{1}$ AND JÁNOS ABONYI *1
${ }^{1}$ MTA-PE Lendület Complex Systems Monitoring Research Group, Department of Process Engineering, University of Pannonia, Egyetem u. 10, Veszprém, H-8200, HUNGARY

\begin{abstract}
Human resources are still utilized in many manufacturing systems, so the development of these processes should also focus on the performance of the operators. The optimization of production systems requires accurate and reliable models. Due to the complexity and uncertainty of the human behavior, the modeling of the operators is a challenging task. Our goal is to develop a worker movement diagram based model that considers the stochastic nature of paced open conveyors. The problem is challenging as the simulator has to handle the open nature of the workstations, which means that the operators can work ahead or try to work off their backlog, and due to the increased flexibility of the moving patterns the possible crossings which could lead to the stopping of the conveyor should also be modeled. The risk of such micro-stoppings is calculated by Monte-Carlo simulation. The applicability of the simulator is demonstrated by a well-documented benchmark problem of a wire-harness production process.
\end{abstract}

Keywords: Industry 4.0, Operator 4.0, Monte-Carlo simulation, Process development, Line balancing, Wire-harness assembly

\section{Introduction}

Conveyor lines are more productive than regular assembly lines [1]; therefore there are more prevalent in the automotive industry [2]. The movement of these conveyors mostly has paced and cyclic characteristic where at the beginning of the cycle, every station moves to the next position [3]. It can happen that the operator cannot finish his/her work before the product leaves the workstation. There are two alternative approaches for completing the unfinished work. We speak about close station production when the operator must stop the conveyor even in case of a minor delay [4]. Such processes are typical in Japan. In U.S.-type production systems, the operator does not have to finish his or her job, he or she can move with the product to the next station to work off the backlog. In these open stations the operators can work ahead or can be delayed [5], and the production stops only when the delay exceeds a critical limit. These open workstations reduce capacity loss by decreasing the risk of stopping the conveyor, but the modeling and optimization of these processes is much more challenging as the model has to handle idle and delay times [6]. Worker movement diagrams are widely used to model the work of operators at conveyor belts [7]. Such models can be used to reduce the risk of conveyor stoppage [8] and optimize production sequence [9], since the optimal distribution of the products can also reduce the probability of critical backlogs [10].

\footnotetext{
*Correspondence: researcher@abonyilab.com
}

Worker movement diagrams focus only one station. In open paced conveyors the operators effect on each other; therefore the model should handle the interactions between the workstations, especially for the prediction of the conveyor stoppage.

Our goal is to develop a worker movement diagram based model for open paced conveyors, which model considers the stochastic nature of production and recognizes the meeting point of operators and analyzes the idle times due to working in the same zones and risk of stopping the production in case of unmanageable backlogs. We introduce stochastic variables into the movement diagram representation based model and apply Monte-Carlo simulation to evaluate the risk of conveyor stoppage and give robust estimates of the effects of different parameter settings. The simulator is developed in Python environment. The applicability of the proposed model and simulator is demonstrated by a well-documented benchmark problem of a wire-harness production process.

Section 2 describes the worker movement diagram and the sections defined based on the relative position of the operators and the conveyor. The model of the paced conveyor is based on equations that represent the movement of the operators in these sections. Based on these equations we calculate when the conveyor should be stopped. Section 3 describes the applicability of the developed simulator in a wire harness production system. 


\section{Model of the paced conveyor}

\subsection{Problem definition}

The most widespread paced open station conveyors are used to produce wire harnesses in the automobile industry. The optimization and cost estimation of these processes are an economically significant problem [11]. These modular assembly lines consist of manual workstations (tables) shown in Fig. 1. Human operators work at the tables that are moving similarly as a conveyor belt (see Fig. 2). These tables move with a fixed speed which is determined based on the tact time, $t_{c}$. After the cycle, every table moves to the next station. The modeling the relative position of the operators and the tables can be represented by worker movement diagrams that will be presented in the next section.

\subsection{Movement diagram of an open station}

The paced conveyor has $k, k=1, \ldots, K$ is number of workstations that moves in every $n=1, \ldots, N$ cycle. The speed of the conveyor is $v_{c}$, and the walking speed of operator is $v_{w}$. The $t_{c}$ is the tact time determines the assembly speed:

$$
v_{n}^{k}=\frac{L}{t_{\pi(n-k)}^{k}}
$$

where $L$ represents the length o the workstation, the $t_{\pi(n-k)}^{k}$ the assembly time which is dependent on the produced product. The sequence of the products is represented by a $\pi$ vector of the labels of the types, so $\pi(k)=p_{j}$ states that type product $p_{j}$ started to be produced during the $k$-th production cycle. The modeling of the paced conveyor is complex task as the conveyor moves only for a $t_{c m}<t_{c}$ period of the time, which defines several sections of the tack time according to the speed and position of the table and the operators.

As it is depicted in Figs. 3 and 4, the worker movement diagram is divided for six sections $(s=1, \ldots, 6)$

1. The operator moves to the starting point of the table

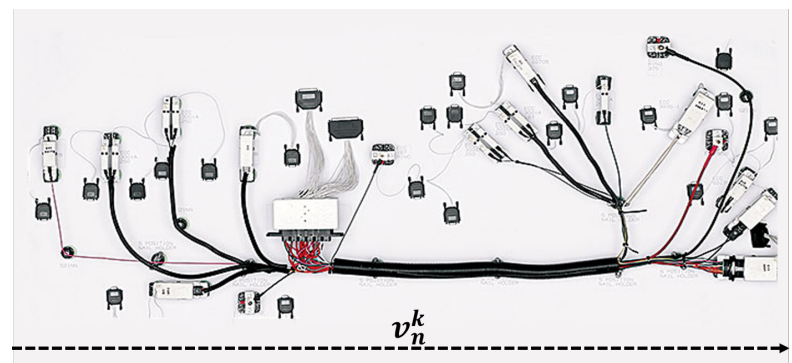

Figure 1: An example assembly table in wire harness manufacturing. The dashed line with an arrow represents the worker motion at the table. The operator works on the table from left to right. The assembly speed is $v_{n}^{k}$.

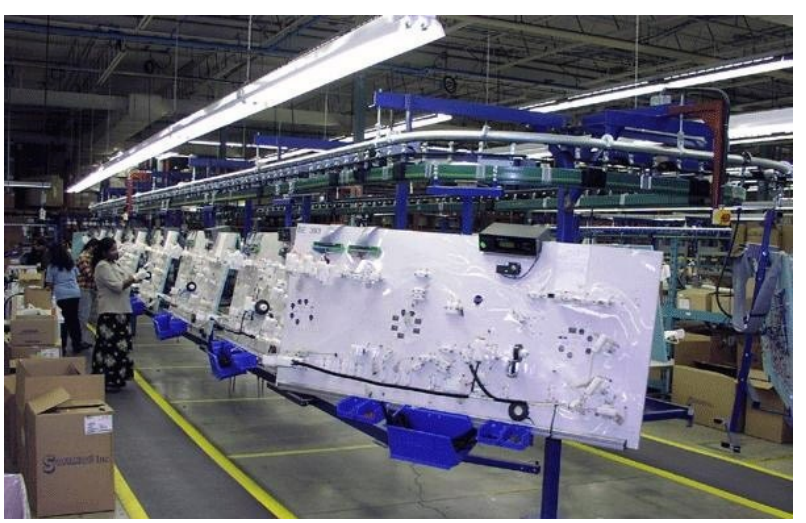

Figure 2: The most widely used open station paced conveyors are used in wire harness assembly, where the operators are working at tables that moving in every cycle of the production [12].

2. The operator works before the new cycle

3. The operator and the table move together

4. The operator works and the table stays

5. The operator and the table move together after the end of tact time

6. The operator works and the table stays after the end of tact time

In the first section, $s=1$, the operator walks to the left side of the table, $F(1)_{n}^{k}$. After reaching this position the operator starts the assembly process and moves with the conveyor till the conveyor moves to its next workspace. After this $F(3)_{n}^{k}$ position the operator works at the standing table with a $v_{n}^{k}$ speed. When the job is finished, the operator reaches the end of the table, $F(4)_{n}^{k}=$ $k L$, as it is shown at Fig. 5). The second, fifth and sixth sections happen when operator deviates from this normal case (work ahead or delayed).

In the following, we present a model that describes how the positions of the table and the operators are changing in time. In the model $F(s)_{n}^{k}$ denotes the position of $k$ th operator at $n$th cycle step in $s$ th section of diagram, where the positions are measured from the starting point of the first table.

\section{Section 1. - The operator moves to the starting point of the table}

At the beginning of the cycle, the operator moves the starting point of the next table which is $2 L$ far from its actual position. The $F(1)_{n}^{k}$ position when the $k$ th operator reaches the staring point of table should be calculated as

$$
\begin{gathered}
F(1)_{n}^{k}=F(6)_{n-1}^{k}-T(1)_{n}^{k} v_{w} \\
T(1)_{n}^{k}=\mathrm{NWT}+\mathrm{DWT}+\mathrm{CDWT}+\mathrm{IWT}
\end{gathered}
$$

where $F(6)_{n-1}^{k}$ is the $k$ th operator finishing position in the previous cycle step $(n-1)$, while the $T(1)_{n}^{k}$ required 


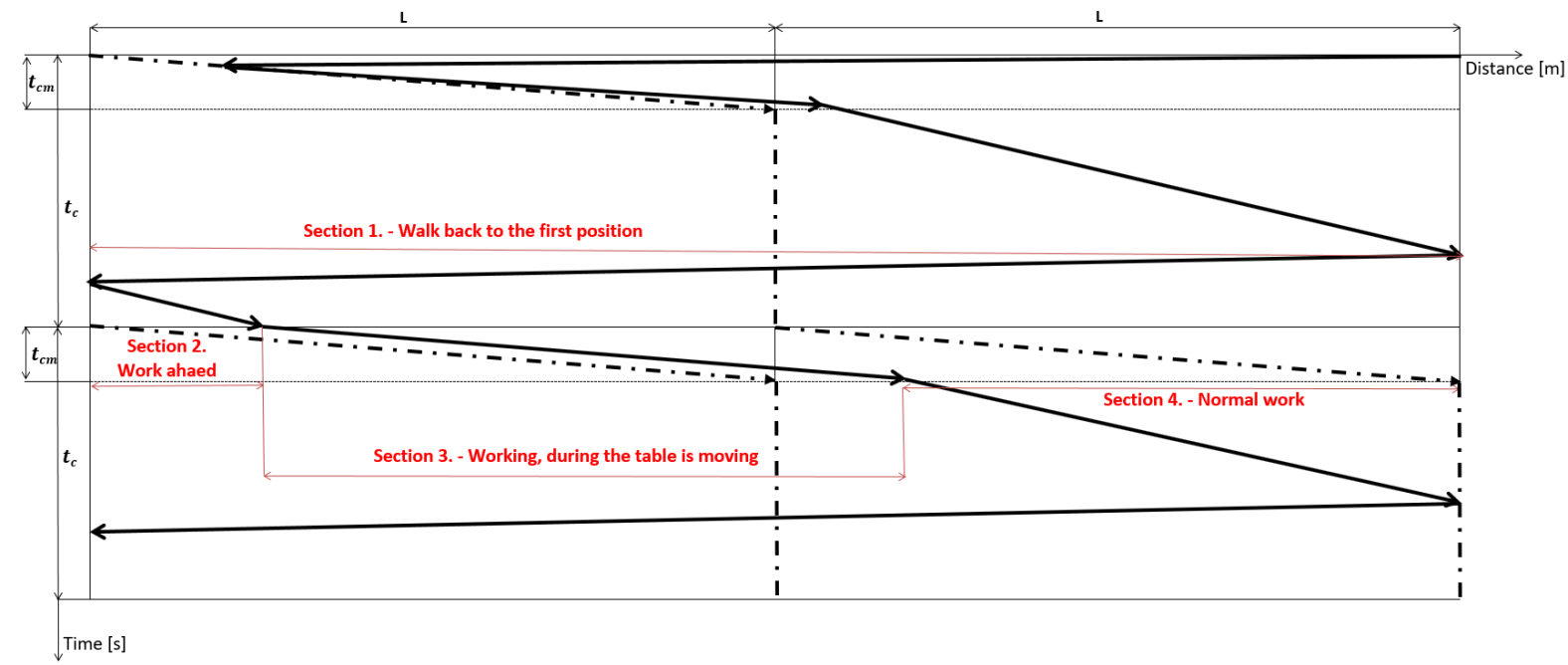

Figure 3: Worker movement diagram of the sections when the operator works ahead. Lines with arrow represent the motion of the operator, while dashed lines represent movement of the table.

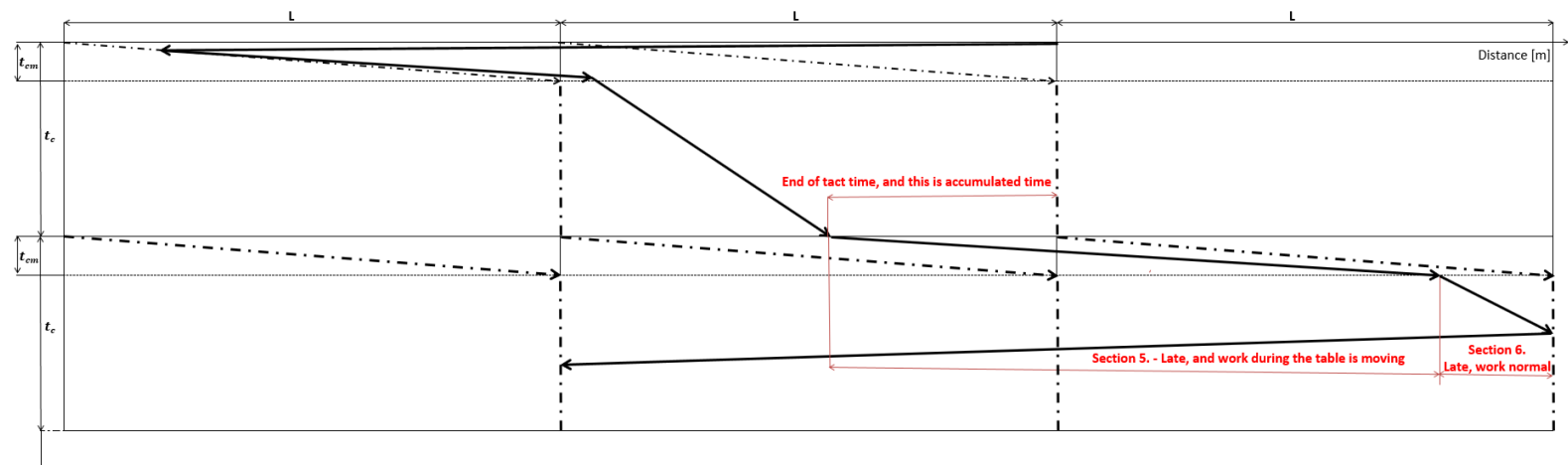

Time [s]

Figure 4: Worker movement diagram of the sections when the operator has a backlog. Lines with arrow represent the motion of the operator, while dashed lines represent movement of the table.

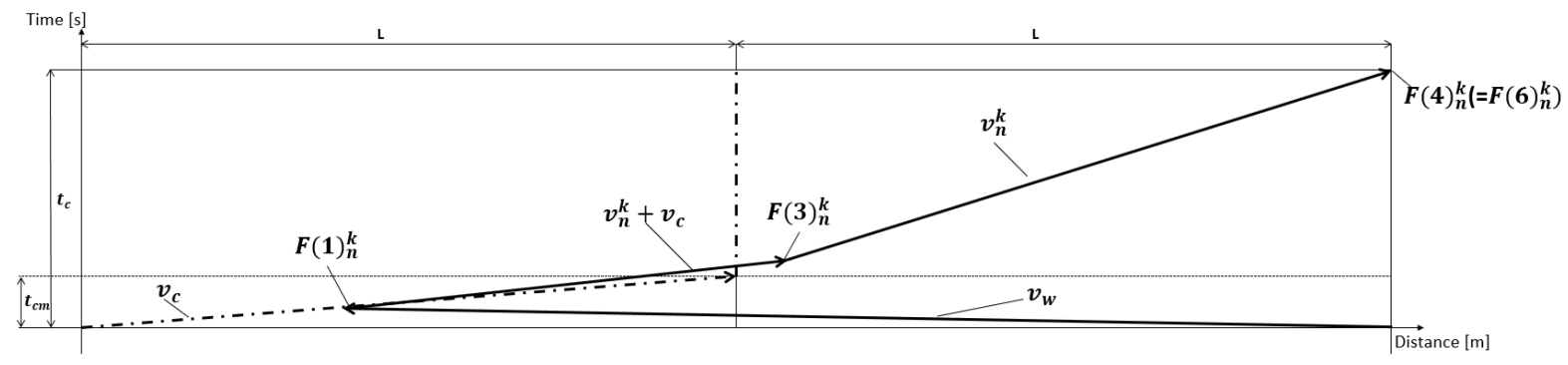

Figure 5: Worker movement diagram of one station. The first meeting point with the table and the operator is $F(1)_{n}^{k} . F(3)_{n}^{k}$ and $F(4)_{n}^{k}$ are the positions at the end of the second and third sections. When there is no delay or the operator does not work ahead $F(4)_{n}^{k}=F(6)_{n}^{k}$, where $F(6)_{n}^{k}$ is the finishing position. 
time can be decomposed into four components, which will be modeled in the following subsections:

- NWT - Normal Walking Time

- DWT - Delayed Walking Time

- CDWT - Critically Delayed Walking Time

- IWT - Idle Walking Time

\section{NWT: Normal Walking Time}

In the normal case, the operator and the conveyor move together at the beginning of the cycle with $v_{c}+v_{w}$ relative speed. The effect of the $a_{n-1}^{k}$ idle time and the $l_{n-1}^{k}$ late time of the previous cycle is represented by the $\mathrm{NWT}_{\mathrm{a}}$ and $\mathrm{NWT}_{\mathrm{b}}$ variables that are used to calculate the NWT walking time:

$$
\begin{aligned}
\mathrm{NWT} & =\max \left[\min \left(\mathrm{NWT}_{\mathrm{a}} ; \mathrm{NWT}_{\mathrm{b}}\right) ; 0\right] \\
\mathrm{NWT}_{\mathrm{a}} & =\max \left(\frac{2 L-a_{n-1}^{k} v_{w}}{v_{c}+v_{w}} ; 0\right) \\
\mathrm{NWT}_{\mathrm{b}} & =\min \left(t_{c m}-l_{n-1}^{k}, \frac{2 L}{v_{c}+v_{w}}\right),
\end{aligned}
$$

where $a_{n-1}^{k} v_{w}$ represents the walking distance of operator at the end of the previous cycle. When $t_{c m}-l_{n-1}^{k}$ is less than zero, then operator does not have to walk, because he or she still works on the last $(n-1)$ product (In this case we should calculate DWT).

\section{DWT: Delayed Walking Time}

When the assembly time in the previous cycle exceeds $t_{c}$, DWT is equal to the time which necessary for the reaching the table after $t_{c}$.

$$
\begin{aligned}
\mathrm{DWT}= & \mathrm{IF}\left[t_{c m}-l_{n-1}^{k}>0 \text { OR } l_{n-1}^{k}=0\right] ; \\
& \text { THEN DWT } ; \text { ELSE } 0
\end{aligned}
$$

$\mathrm{DWT}_{\mathrm{h}}=$

$\max \quad\left[\frac{2 L}{v_{c}+v_{w}}-\max \left(t_{c m}-l_{n-1}^{k} ; 0\right) ; 0\right] v_{c w}$

where $v_{c w}=\frac{v_{c}+v_{w}}{v_{w}}$ is the walking speed of operator, when the conveyor is moving.

\section{CDWT: Critically Delayed Walking Time}

When $l_{n-1}^{k}$ is more than $t_{c m}$, the operator moves to the beginning of the table when the conveyor is standing.

$$
\begin{aligned}
\mathrm{CDWT}= & \mathrm{IF}\left[t_{c m}-l_{n-1}^{k}<=0\right. \\
& \text { OR } \left.l_{n-1}^{k}=0\right] ; \\
& \text { THEN } \frac{2 L}{v_{w}} ; \text { ELSE } 0
\end{aligned}
$$

\section{IWT: Idle Walking Time}

When the conveyor does not move and $a_{n-1}^{k}$ is bigger than the necessary walking time, $\frac{2 L}{v_{w}}$, then

$$
\mathrm{IWT}=\min \left(a_{n-1}^{k}, \frac{2 L}{v_{w}}\right)
$$

\section{Section 2. - The operator works before the new cycle}

The $F(2)_{n}^{k}$ staring position and $T(2)_{n}^{k}$ duration of the The second section is calculated as:

$$
\begin{aligned}
F(2)_{n}^{k} & =F(1)_{n}^{k}+T(2)_{n}^{k} v_{n}^{k} \\
T(2)_{n}^{k} & =\max \left(a_{n-1}^{k}-\frac{2 L}{v_{w}} ; 0\right)
\end{aligned}
$$

where $v_{n}^{k}$ is the average speed of the assembly.

\section{Section 3. - The operator and table move together}

In this section, operator and the conveyor are moving together for a time period shorter than $t_{c} m$, so they will meet at:

$$
\begin{array}{r}
F(3)_{n}^{k}=F(2)_{n}^{k}+T(3)_{n}^{k}\left(v_{c}+v_{n}^{k}\right) \\
T(3)_{n}^{k}=\min \left(\max \left[(n-1) t_{c}+t_{c m}-T^{k} ; 0\right] ; t_{c m}\right)
\end{array}
$$

where $(n-1) t_{c}+t_{c m}$ describes the time instant the section will finish. In normal situation $T(3)_{n}^{k}$ equals to $t_{c m}$, while in extreme case the operator has as significant idle time as he or she finishes his or her job before the end of this section.

\section{Section 4. - The operator works and table stays}

In this section the operator works with $v_{n}^{k}$ linear speed until the conveyor does not move, so this section finishes at:

$$
\begin{aligned}
F(4)_{n}^{k}= & F(3)_{n}^{k}+T(4)_{n}^{k} v_{n}^{k} \\
T(4)_{n}^{k}= & \min \left\{\max \left[n t_{c}-T ; 0\right]\right. \\
& \left.\frac{L}{v_{n}^{k}}-T(2)_{n}^{k}-T(3)_{n}^{k}\right\}
\end{aligned}
$$

where $\frac{L}{v_{n}^{k}}-T(2)_{n}^{k}-T(3)_{n}^{k}$ defines the remaining assembly time before the end of the tact time.

\section{The idle and delay times}

At the end of the cycles the $a_{n}^{k}$ idle or $l_{n}^{k}$ delay time is calculated as:

$$
\begin{aligned}
& l_{n}^{k}=\max \left(\frac{L}{v_{n}^{k}}+T(1)_{n}^{k}-a_{n-1}^{k}+l_{n-1}^{k}-t_{c} ; 0\right)(17) \\
& a_{n}^{k}=\max \left(t_{c}-\frac{L}{v_{n}^{k}}-T(1)_{n}^{k}+a_{n-1}^{k}-l_{n-1}^{k} ; 0\right)(18)
\end{aligned}
$$

The prediction of conveyor stoppage is the most important ability of the model which will be calculated based on the delay time as it will be presented in the following subsection. 


\section{Section 5. - The operator and the table move together after the end of tact time}

This section can be considered as the modification of the third section with the delay of the operator. As we already know $l_{n}^{k}$ and the operator can work in this section maximum till $t_{c m}$, the calculation is straightforward:

$$
\begin{aligned}
F(5)_{n}^{k} & =F(4)_{n}^{k}+T(5)_{n}^{k}\left(v_{c}+v_{n}^{k}\right) \\
T(5)_{n}^{k} & =\min \left(l_{n}^{k}, t_{c m}\right)
\end{aligned}
$$

\section{Section 6. - The operator works and the conveyor stays after the end of tact time}

As the duration of this section is limited as $t_{c}-t_{c m}$, the variables that define the end of the section are calculated as:

$$
\begin{aligned}
F(6)_{n}^{k} & =F(5)_{n}^{k}+T(6)_{n}^{k} v_{n}^{k} \\
T(6)_{n}^{k} & =\min \left[\max \left(l_{n}^{k}-t_{c m} ; 0\right) ; t_{c}-t_{c m}\right]
\end{aligned}
$$

\section{Calculation of the stoppage and the idle time}

The open station type operation of the paced conveyor has increased flexibility as the conveyor has to be stopped only when the delay of the $k$ th operator is as significant as it disturbs the work of the neighboring $k-1$ th operator. We define this situation as:

$$
T^{k}+T(1)_{n}^{k}<=T^{k-1}+T(1)_{n-1}^{k-1}+\frac{t_{c}}{4}
$$

In this case the idle $I_{n}^{k}$ time has to be modified by $T(1)_{n}^{k}$ and reset the value of $a_{n}^{k}$ to zero.

$$
I_{n}^{k}=(n-1) t_{c}-T(1)_{n}^{k}
$$

When the $l_{n}^{k}-T(6)_{n}^{k}$ is smaller than $t_{c m}$, the operator stops the conveyor. The $l_{n}^{k}$ is reset and the stoppage time is:

$$
S_{n}^{k}=\max \left(l_{n}^{k}-T(6)_{n}^{k}-t_{c m} ; 0\right)
$$

\subsection{KPls and the developed simulator}

The developed simulator handles the stochastic and open nature of the conveyor, simulates all workstations, the interactions between the operators and predicts stoppage. The worker movement diagram representation helps in the stoppages prediction (see Fig. 6).

Production planners can use the developed simulator to try sequencing strategies and analyze a new production lines capability. The following key performance indicators (KPIs) calculated based on Monte-Carlo simulation gives a realistic picture about the production.

- The balance of conveyor line is depended on the maximum of the late times of operators, $\mathbf{l}^{k}=$ $\left[l_{1}^{k}, l_{2}^{k}, \ldots, l_{n}^{k}\right]$ :

$$
B=\frac{\sum_{k=1}^{K} \max \left(\mathbf{l}^{k}\right)}{K} \frac{1}{t_{c}}
$$

- The efficiency of production is calculated based on to the sum of the $\frac{L}{v_{n}^{k}}$ assembly times divided by the maximum of the $\mathbf{T}(6)^{k}=$ $\left.T(6)_{1}^{k}, T(6)_{2}^{k}, \ldots, T(6)_{n}^{k}\right)$ finishing times and the sum of stoppage times multiplied by the number of workstations.

$$
P=\frac{\sum_{k=1}^{K} \frac{L}{v_{n}^{k}}}{\left\{\max \left[\mathbf{T}(6)^{k}\right]+\sum_{n=1}^{N} \sum_{k=1}^{K} S_{n}^{k}\right\} K}
$$

- The sum of the $S$ stoppage times (Eq. 25).

- The mean of the assembly times.

The simulator and the movement diagram are developed in Python environment. $\mathrm{d}$ The developed simulator and the related dataset is freely and fully available on the website of authors: www. abonyilab.com.

\section{Application to wire harness production}

To demonstrate the applicability of the simulator three typical types of production sequencing strategies were analyzed. In the first case, the sequence follows the random customer demand which case often happens in Just In Time (JIT) production. Batch production is a more efficient sequencing strategy. In this case, batches of lower and higher complexity products are following each other. One of the best solutions is the $\pi=m_{1}, m_{2}, m_{1}, \ldots$ high/low sequencing strategy because it utilizes the open station nature of the conveyor.

The studied conveyor contains $K=5$ workstations. The number of manufactured products is $N=100$, and two different group of products $(M=2)$ are produced. The assembly times are represented by a normal distribution, which is $t_{1}=\mathcal{N}(250,30)$ for the lower complexity product and $t_{2}=\mathcal{N}(310,30)$ for the higher complexity product. The tact time of the conveyor is constant and set to $t_{c}=280 \mathrm{~s}$ which is the average assembly time of the products.

Fig. 7 shows the results of 1,000 simulations of the three sequence types. This scatter matrix plot shows the main KPIs, the balance, the number of the manufactured products, the number of stoppages, and the average assembly times. The green dots represent the High/Low, the blues the batched, and the red the random sequences.

As shown in Fig. 7, the difference between the random (blue) and high/low (green) sequences is significant on all KPIs. The batched sequence (red) has similar performance to the high/low sequence, but many times this batch production is not manageable because of the high variance of the products and the short delivery times.

\section{Conclusions}

As human resources are still necessary for many manufacturing systems, the development of production process should also focus on the performance of operators. 


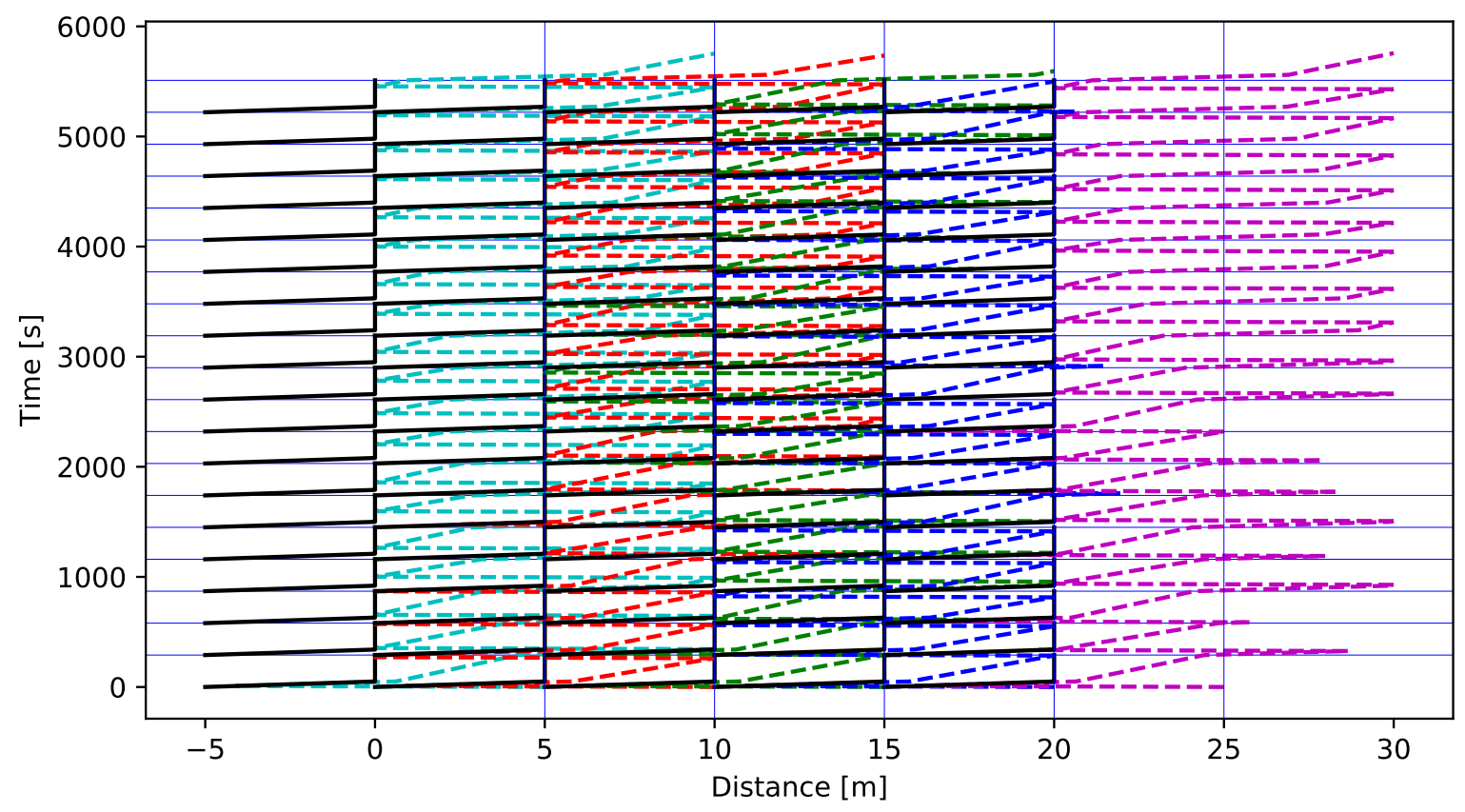

Figure 6: The developed worker movement diagram of five stations and 18 cycle steps. The distance begins at $-5 \mathrm{~m}$ to represent the previous workstation.
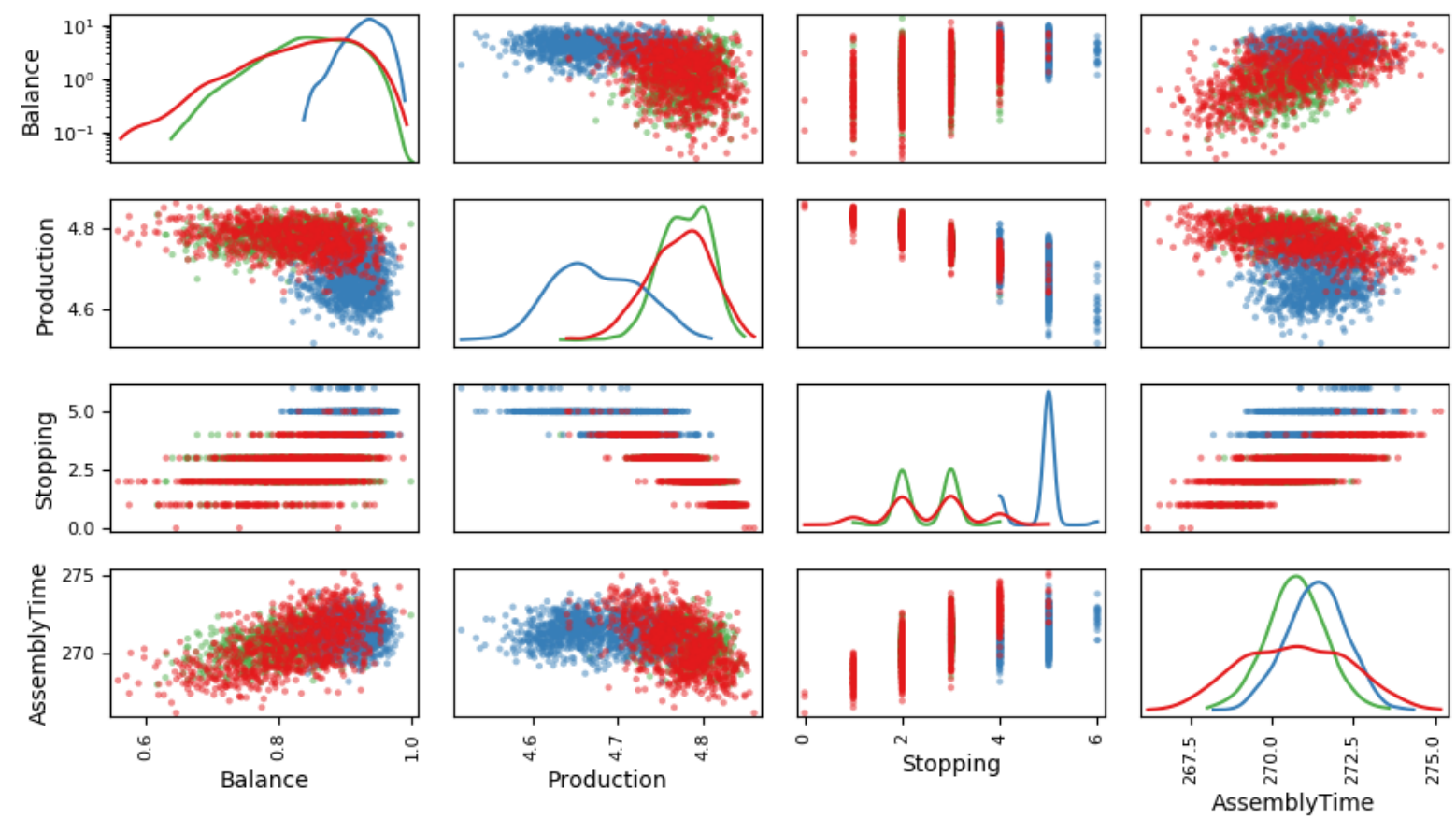

Figure 7: The result of a Monte-Caro simulation of three different sequencing strategies. The scatter plot shows all of KPIs. The high/low sequence is denoted by green, the batch by red, and the random by blue dots. The difference between the random and high/low sequences is significant on all KPIs. 
According to the digital twin concept, this development should be based on the model of the production system, which necessaries the development of simulators that can handle uncertainties related to the human nature of the operators. The developed worker movement diagram based model handles the paced and open workstations of the conveyors and the stochastic nature of production. The worker movement representation helps in the analysis of the operators which is needed to predict production stoppages. The introduction of stochastic variables and the Monte-Carlo simulation-based evaluation of the key performance indicators provide a realistic picture about the production. The applicability of the simulator in the analysis of the effect of production sequencing is demonstrated by a well-documented benchmark problem of a wire-harness production process. The developed simulator is not specialized to the studied wire harness production; it can be used to model all of the types of paced conveyors even open or closed workstations.

\section{Acknowledgement}

This research was supported by the National Research, Development and Innovation Office NKFIH, through the project OTKA-116674 (Process mining and deep learning in the natural sciences and process development) and the EFOP-3.6.1- 16-2016- 00015 Smart Specialization Strategy (S3) Comprehensive Institutional Development Program.

\section{Notations}

\begin{tabular}{|c|c|}
\hline KPI & Key Performance Indicator \\
\hline NWT & Normal Walking Time \\
\hline DWT & Delayed Walking Time \\
\hline CDWT & Critically Delayed Walking Time \\
\hline IWT & Idle Walking Time \\
\hline$k$ & index of workstation $k=1, \ldots, K$ \\
\hline$K$ & number of workstations \\
\hline$n$ & index of cycle step $n=1, \ldots, N$ \\
\hline$N$ & number of cycle steps \\
\hline$s$ & index of section $s=1, \ldots, 6$ \\
\hline$v_{n}^{k}$ & assembly speed of th operator $\left[\frac{\mathrm{m}}{\mathrm{s}}\right]$ \\
\hline$v_{w}$ & walking speed of the operator $\left[\frac{\mathrm{m}}{\mathrm{s}}\right]$ \\
\hline$v_{c}$ & speed of the conveyor $\left[\frac{\mathrm{m}}{\mathrm{s}}\right]$ \\
\hline$v_{c w}$ & $\begin{array}{l}\text { walking speed of the operator when } \\
\text { the conveyor is moving }\left[\frac{\mathrm{m}}{\mathrm{s}}\right]\end{array}$ \\
\hline$t_{c}$ & tact time $[\mathrm{s}]$ \\
\hline$t_{c m}$ & conveyor movement time $[\mathrm{s}]$ \\
\hline$t_{(\pi(n)}^{k}$ & $\begin{array}{l}\text { assembly time of actual the } \\
\text { product at } k \text { th operator }[\mathrm{s}]\end{array}$ \\
\hline$T(s)_{n}^{k}$ & $\begin{array}{l}\text { duration of the actual } s \text { section in } n \text {th cycle } \\
\text { step a } k \text { th workstation }[\mathrm{s}]\end{array}$ \\
\hline $\mathbf{T}(6)^{k}$ & finishing times of the $k$ th operator $[\mathrm{s}]$ \\
\hline$a_{n}^{k}$ & $\begin{array}{l}\text { work ahead time in } n \text {th cycle step at } \\
k \text { th operator }[\mathrm{s}]\end{array}$ \\
\hline$l_{n}^{k}$ & late time in $n$th cycle step at $k$ th operator [ \\
\hline
\end{tabular}

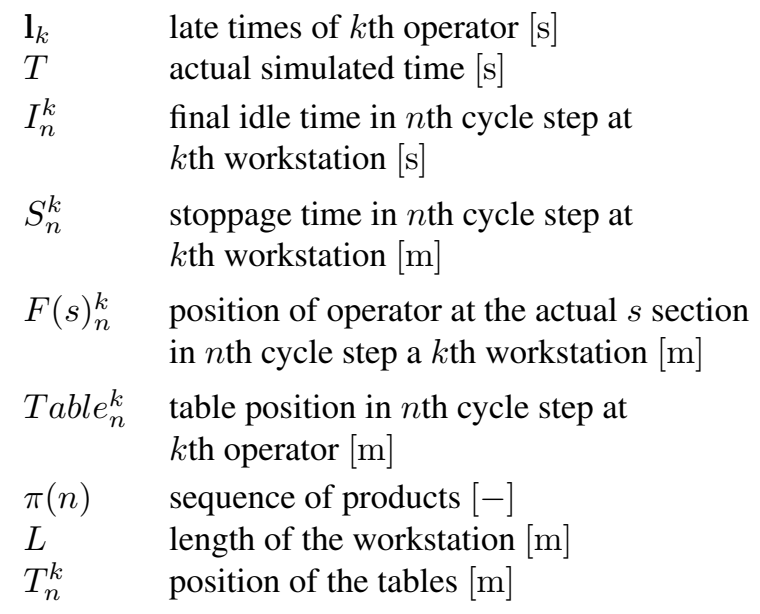

\section{REFERENCES}

[1] Estrada, F., Villalobos, J.R., Roderick, L., Estrada, F., Villalobos, J.R., Roderick, L.: Evaluation of JustIn-Time alternatives in the electric wire-harness industry, Taylor and Francis, 1997 35(7), 1993-2008, DOI: 10.1080/002075497195038

[2] Lodewijks, G.: Two Decades Dynamics of Belt Conveyor Systems Bulk Solids Handling, 2002 22(2), 1-2

[3] Xiaobo, Z., Zhou, Z., Asres, A.: Note on Toyota's goal of sequencing mixed models on an assembly line, Computers and Industrial Engineering, 1999 36(1), DOI: 10.1016/S0360-8352(98)00113-2, 57-65

[4] Sarker, B.R., Pan, H.: Designing a Mixed-Model, Open-Station Assembly Line Using Mixed-Integer Programming, The Journal of the Operational Research Society Palgrave Macmillan Journals, 2001 52(52), 545-558 DOI: 10.1057/palgrave.jors. 2601118

[5] Bukchin, J., Tzur, M.: Design of flexible assembly line to minimize equipment cost, IIE Transactions (Institute of Industrial Engineers), 2000 32(7), 585598 DOI: 10.1080/07408170008967418

[6] Bautista, J., Cano, J.: Minimizing work overload in mixed-model assembly lines, International Journal of Production Economics, 2008 112(1), 177-191 DOI: 10.1016/j.ijpe.2006.08.019

[7] Xiaobo, Z., Ohno, K.: Algorithms for sequencing mixed models on an assembly line in a JIT production system, Computers ind. Engng, 1997 32(1), 47-56 DOI: 10.1016/s0360-8352(96)00193-3

[8] Xiaobo, Z., Ohno, K.: Properties of a sequencing problem for a mixed model assembly line with conveyor stoppages, European Journal of Operational Research, 2000 124(3), 560-570 DOI: 10.1016/S03772217(99)00198-8

[9] Fattahi, P., Salehi, M.: Sequencing the mixed-model assembly line to minimize the total utility and idle costs with variable launching interval, International Journal of Advanced Manufacturing Technology, 2009 45(9-10), 987 DOI: 10.1007/s00170-009-2020-0 
[10] Tsai, L.H.: Mixed-Model Sequencing to Minimize Utility Work and the Risk of Conveyor Stoppage Mixed-model Sequencing to Minimize Utility Work and the Risk of Conveyor Stoppage, Source: Management Science, 1995 41(3), 485-495 DOI: 10.1287/mnsc.41.3.485
[11] Ong, N.S., Boothroyd, G.: Assembly times for electrical connections and wire harnesses, The International Journal of Advanced Manufacturing Technology, 1991 6(2), 155-179 DOI: 10.1007/BF02601438

[12] Assembly Line Conveyor Systems, 2015, https: //www.pacline.com/photos/photos-by-solution/ assembly-line-conveyors/ 